\title{
Personalisation of Context-Aware Solutions Supporting Asthma Management
}

\author{
Mario Quinde ${ }^{1,2(\bowtie)}$, Nawaz Khan ${ }^{1}$, and Juan Carlos Augusto ${ }^{1}$ \\ 1 Research Group on Development of Intelligent Environments, \\ Middlesex University, London, UK \\ MQ093@live.mdx.ac.uk, \{N.X.Khan, J.Augusto\}@mdx.ac.uk \\ 2 Universidad de Piura, Piura, Peru \\ mario.quinde@udep.pe
}

\begin{abstract}
Personalisation of asthma management plans is important because asthma patients experience different triggers and symptoms as a result of the high heterogeneity level of the condition. Although this makes context-awareness suitable to support asthma management, existing context-aware solutions do not allow patients to personalise their management plans. This research proposes an approach to develop context-aware solutions allowing the personalisation of asthma management plans. It is derived on the basis of the literature review and a qualitative research that includes both asthma patients and carers. A prototype to illustrate the application of the approach is demonstrated.
\end{abstract}

Keywords: Mobile health $\cdot$ Asthma $\cdot$ Personalisation Context-awareness

\section{Introduction}

Asthma is a heterogeneous disease characterized by chronic airway inflammation. A patient's asthma is defined by his/her history of airflow limitation and respiratory symptoms (such as wheeze, shortness of breath, chest tightness and cough) that are variable over time and in intensity [1]. Although there is no cure, asthma can be controlled with a treatment based on a management plan to avoid triggers and reduce symptoms [2-4].

Hospitalizations and deaths from asthma have declined in some countries but its prevalence is increasing in many [1]. In 2015 in the US, 24.6 million people had asthma, 11.5 million suffered one or more attacks and 3 thousand died because of it [5]. In 2015 in the UK, 5.4 million people had asthma, every $10 \mathrm{~s}$ someone suffered a potentially life-threatening attack and the annual cost to the NHS of treating asthma was more than $£ 1$ billion [6].

The heterogeneity of asthma is challenging when defining treatments as triggers and symptoms are different to each patient. This makes personalisation

(C) The Author(s) 2018

K. Miesenberger and G. Kouroupetroglou (Eds.): ICCHP 2018, LNCS 10897, pp. 510-519, 2018.

https://doi.org/10.1007/978-3-319-94274-2_75 
important because each patient assigns different priorities to the indicators framing his/her condition. E.g. a patient whose asthma is triggered by low temperatures is more concern about the weather than a patient only allergic to pollen.

From this perspective, context-aware solutions can aid the personalisation of the asthma management process. A context-aware solution "uses context to provide task-relevant information and/or services to a user", where context is "any information that can be used to characterize the situation of an entity, where an entity can be a person, place, or physical or computational object" [7]. Hence, a context-aware solution supporting asthma management should provide information and/or services considering the indicators defining the patient's condition.

This research proposes an approach to develop context-aware solutions aiding the personalisation of asthma management plans. This research attempts to determine the existing gaps of using context-awareness in asthma management and set out the functionalities of such context-aware system based on a qualitative research. This article is divided as follows: Sect. 2 shows the state of the art, Sect. 3 explains the methodology, Sect. 4 describes the proposal and prototype, and Sect. 5 presents the Discussion and Conclusions.

\section{State of the Art}

The application fields of context-awareness, as a component of Intelligent Environments, can be linked to Transportation, Education, Production Places, Smart Offices, Intelligent Supermarkets, Energy Conservation, Entertainment and Health [8]. Examples of context-aware solutions supporting health care are Medication Assistant [9], Hefestos [10] and SOSPhone [11]. Context-awareness has also shown potential to improve management of chronic diseases. An example is a system aiding patients' technique when measuring blood pressure [12]. Another is a system collecting blood-glucose values from diabetes patients to determine their health status and perform actions according to this status [13].

Research works about solutions supporting asthma were reviewed. After an extensive review, eight works were selected as they offer or suggest the use of context-aware features. Table 1 shows the wide variety of indicators tracked by these solutions, which is associated to the high heterogeneity level of asthma. The context-aware features of the researches are linked to: recognizing symptoms and/or triggers $[15,17,20]$, notifying asthma patients and carers when health status is abnormal $[14,16,17,19,20]$, showing near hospitals in emergencies [16], reminding patients to take readings [19], providing advice regarding patients' status [20], and predicting asthma attacks based on patients' context [21].

Although personalisation is the key to implement asthma plans, none of these works allows users to choose the indicators to track, nor the features to use for supporting the management of their condition. The researches do not support a comprehensive framing of context including indicators of several indoor and outdoor places of interest (e.g. home, workplace, path to work). This research aims to close this gap by proposing an approach to develop context-aware solutions allowing the personalisation of asthma management plans and providing a more comprehensive way of framing patients' context to aid in decision-making. 
Table 1. Indicators tracked by the reviewed solutions

\begin{tabular}{l|l|l}
\hline Research & Patient's indicators & Environmental indicators \\
\hline$[14]$ & Medication, control level & - \\
\hline$[15]$ & PEF & Overall air quality \\
\hline$[16]$ & PEF, control level & Sand storms \\
\hline$[17]$ & Wheezing, motion & - \\
\hline$[18]$ & PEF, FEV1, FEV6, NO, CO, O2 & - \\
\hline$[19]$ & NO, control level & Temperature, humidity, CO \\
\hline$[20]$ & $\begin{array}{l}\text { PEF, FEV1, FVC,O2 level, } \\
\text { wheezing, exercise, medication }\end{array}$ & $\begin{array}{l}\text { Temperature, humidity, CO, } \\
\text { O3, NO2, Cl }\end{array}$ \\
\hline$[21]$ & $\begin{array}{l}\text { PEF, FEV1, FVC, heart rate, } \\
\text { respiratory rate, wheezing, motion }\end{array}$ & Temperature, humidity, O3, VOC \\
\hline
\end{tabular}

\section{Methodology}

The User-Centred Intelligent Environments Development Process (U-CIEDP) led the research, focusing on its first primary stage: Initial Scoping. The U-CIEDP suggests to engage stakholders in the scoping, development and installation of Intelligent Environments. It has been used to co-create smart technology for people with special needs [22].

The qualitative research included 4 asthma patients, 2 carers of asthma patients and 1 physician expert in respiratory diseases. The interview was the research method chosen because context-awareness is complex to explain through a questionnaire. Each interview lasted between 40-60 min. It included an explanation of context-awareness before asking a set of open questions to know the indicators they would like to monitor and the features they desire from a contextaware solution supporting asthma management. The interviews were held in the Smart Spaces Lab of Middlesex University.

A prototype is demonstrated as an example of using the approach to create context-aware solutions supporting the personalisation of asthma management. It is under development and uses sensors from the Smart Spaces Lab to monitor indoor environmental factors. Its functionalities are shown in Sect.4.1.

\section{Personalisation of Context-Aware Solutions for Asthma}

The interviews re-confirm the heterogeneity of asthma. Participants stated the variables to track depend on each patient's specific triggers and symptoms. The expected context-aware features are also linked to the patient's contexts. For instance, while a participant said he "wants to be reminded to open the windows when the indoor air quality is not good", another said he "would like to be reminded to close the windows if the forecast says that temperature will drop." Most participants said carers should be notified in emergencies and when 
patient's status is abnormal. They affirmed the notifications should be personalised depending on the patient's scenario. For example, "The solution should notify parents if the patient is a child, and the partner if the patient is an adult..."

The proposal focuses on identifying and classifying the indicators framing the context of a patient to facilitate the personalisation of asthma management plans. It facilitates the description and it provides a more comprehensive support for decision-making. The indicators are classified into Patient's indicators, Indoor Environmental Indicators (IEIs) and Outdoor Environmental Indicators (OEIs) (Fig. 1).

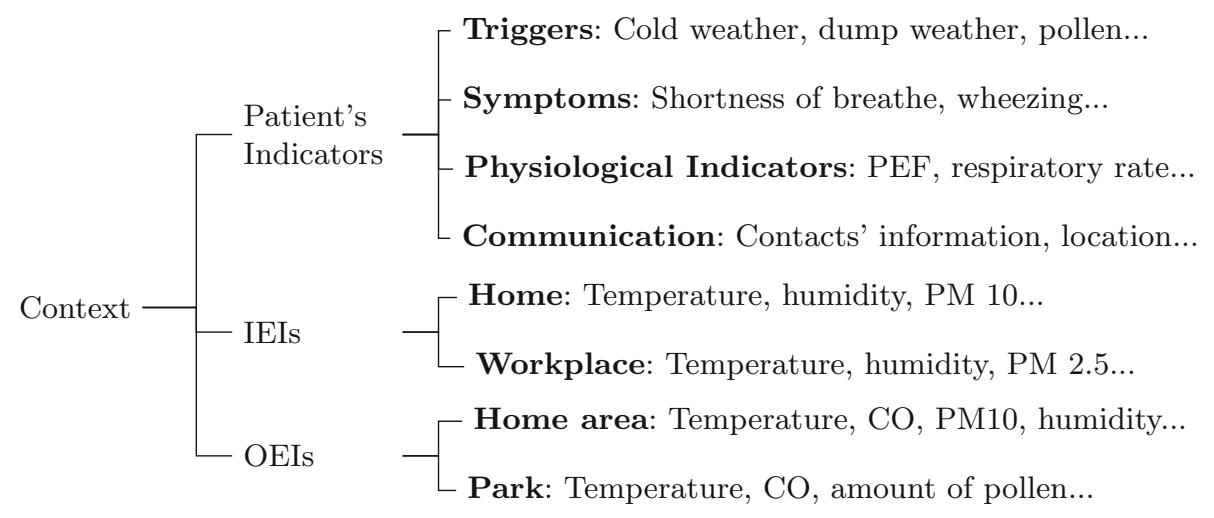

Fig. 1. Classification and examples of indicators framing patient's context

Patient's indicators include triggers and symptoms characterizing the patient's asthma, physiological indicators regarding his/her status and communication information. For instance, a person can choose cold weather and pollen as triggers, shortness of breathe and breathing faster as symptoms, Peak Expiratory Flow (PEF) and heart rate as physiological indicators, and his/her spouse contact information to define the communication component.

IEIs and OIEs define the status of places the patient is interested on. This status is linked to the external factors triggering patient's asthma. These indicators show how exposed the patient would to these factors if $\mathrm{s} /$ he is in those places. IEIs are linked to indoor places and OEIs are linked to outdoor places. Following the previous example, the patient would choose to monitor temperature and PM 2.5 at home and workplace (IEIs) as s/he is sensitive to cold weather and pollen. S/he would also choose to monitor temperature and pollen level in the area nearby home or at a park s/he frequents (OEIs).

One of the reasons to separate indoor and outdoor places is the sources used to get the data. IEIs data tends to be obtained from sensors. OEIs data is usually gathered through APIs (e.g. Google Awareness). It is important to clarify that it is possible to monitor a set of IEIs that is partially or totally different to the set of OEIs. It is even possible to have IEIs (or OEIs) varying from place to place. 
Figure 2 shows the proposed architecture allowing the personalisation of context-aware solutions supporting asthma management. The Personalisation Module alters the Database (DB) according to the personalisation settings chosen by the user. The Report Generator gets these settings and values from the DB to generate the reports for users. The Data Handler gets values from the Data Collectors and inserts it into the DB. The Context-Aware Reasoner (CAR) gets values from the DB to analyse patient's personalised context. Finally the Notification Engine send notifications to users when the context is meaningful.

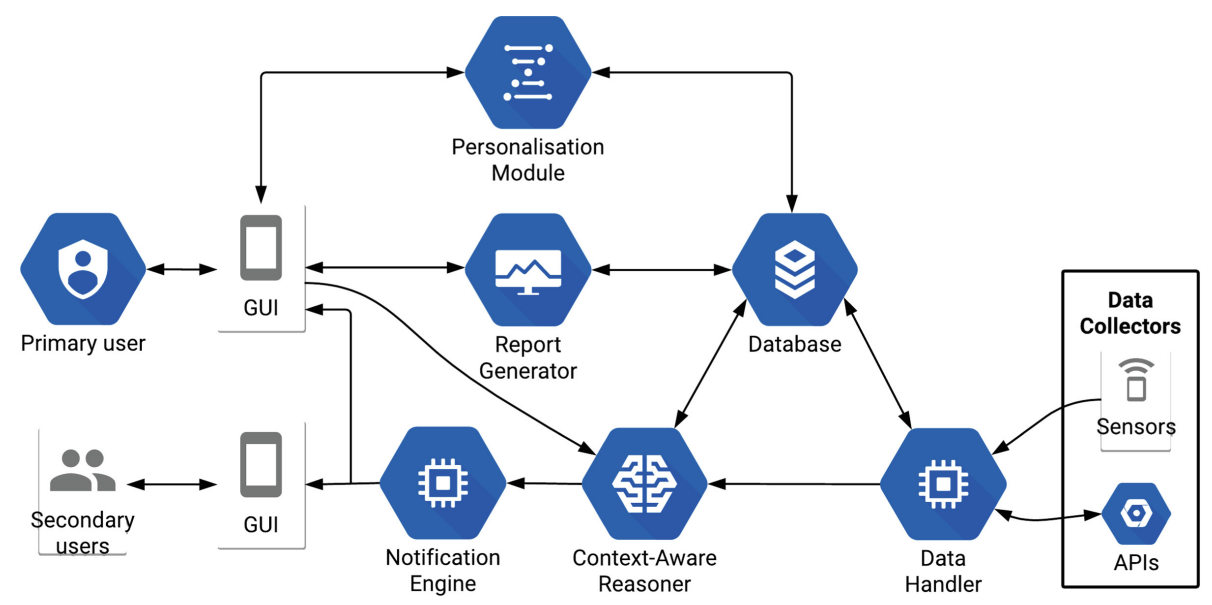

Fig. 2. System architecture

\subsection{Prototype}

The prototype aims to clarify the use of the approach. It is an under development mobile application allowing users to set the indicators they want to track according to the specific characteristics of their asthma. The option "Personalise your Asthma" from the main screen (Fig. 3a) leads to the screen shown in Fig. 3b, which shows the personalisation set by the user. The settings can be changed through the options menu of the screen. An example of how to personalise the indicators to track is shown in Fig. 3c, where user can set the indicators for HOME and establish the control values (MIN,MAX). Users can also choose their triggers, symptoms and the people to contact in case of emergency.

The option "Check your Indicators" (Fig. 3a) allows users to see the values of the indicators they chose. "Deploy Emergency Notifications" automatically sends SMS and emails to the people chosen to be contacted. It also shows a notification on their phones if they have the application installed (Fig. 3d).

The sequence diagram explaining how the fully functional prototype will work is shown in Fig. 4. When the user personalises (1.1), the GUI sends the 


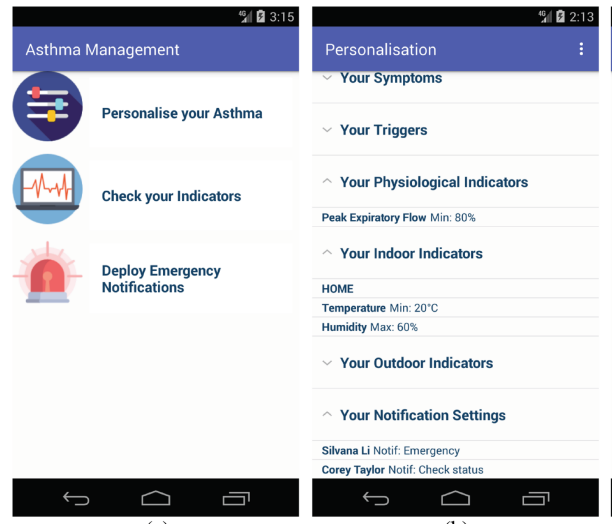

(a)

(b)

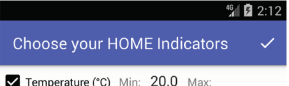

V Temperature (c)) Min: 20.0 Max.

D Humidity (5) Min: $\quad$ Max: 60.0

$\square$ PM 2.5 Min: $\quad$ Max:

$\square$ PM 10 Min: Max:

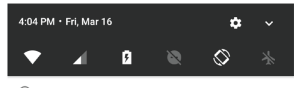

(1) Astima Maragenentr. now

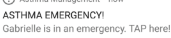

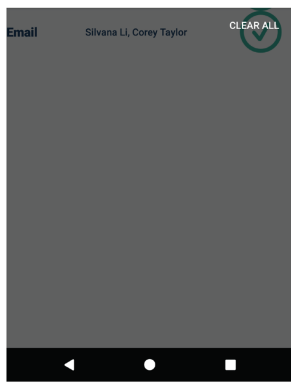

(d)

Fig. 3. Screens of the prototype

Sequence diagram: pull approach

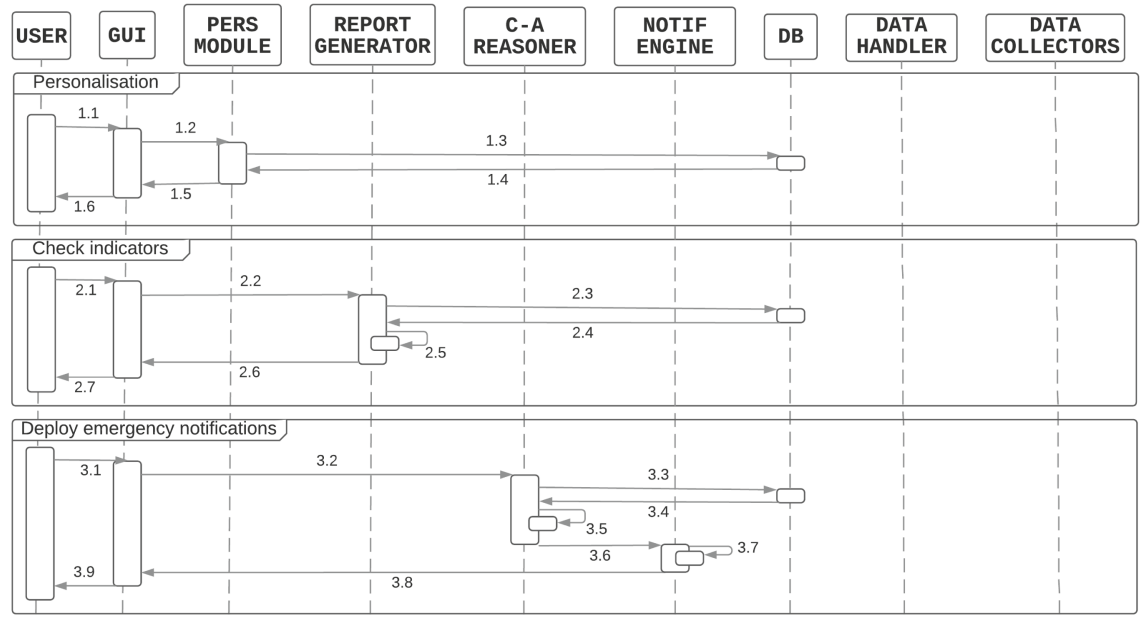

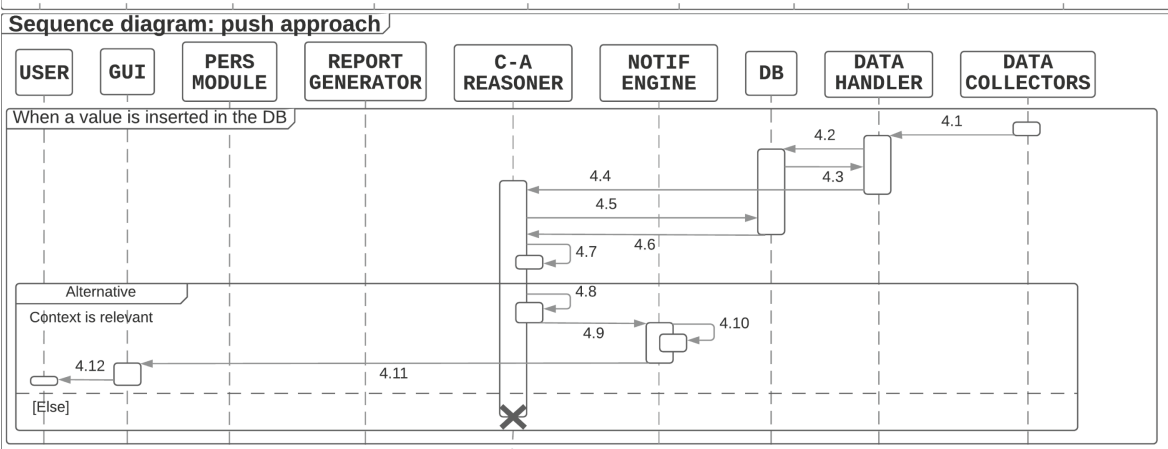

Fig. 4. Sequence diagram 
settings to the Pers. Module (1.2), which modify the DB according to these settings (1.3). The DB replies with a confirmation message that goes to the user (1.4-1.6). When the user chooses to check the status of the indicators (2.1), the Report Generator is activated (2.2). It gets the personalisation settings and the indicators values from the DB $(2.3,2.4)$ to prepare the report $(2.5)$ that is shown to the user $(2.6,2.7)$. When the emergency notification feature is chosen (3.1), the CAR is activated (3.2). It gets the data from the DB $(3.3,3.4)$ to prepare the context data (3.5) that is delivered to the Notif Engine (3.6), which sends the emails and SMSs to the people chosen by the user and notifies them through the mobile application (3.7). Then, it sends a confirmation to the user $(3.8,3.9)$.

The system also automatically notifies users when there is a hazard. For this, the Data Handler gets data from the Data Collectors (4.1), stores it into the DB $(4.2,4.3)$ and activates the CAR (4.4). The CAR gets data from the DB (4.5, 4.6) to analyse the context (4.7) according to the personalisation settings. If the context is relevant, the CAR prepares the context data (4.8) that is sent to the Notif Engine (4.9), which delivers notifications (through the app) to the people chosen by the user (4.10) and notifies the user regarding the hazard(s) detected $(4.11,4.12)$. If the context is not relevant, the CAR terminates its activity.

\section{Discussion and Conclusions}

The research proposes an approach to personalise context-aware solution supporting asthma management. The main contribution is to combine patient's indicators, IEIs and OEIs to frame the context of a patient. The proposal enables the users to accommodate their own scenarios, hence, allowing the personalisation.

Tracking the right variables is important given heterogeneity of patients [24]. Moreover, if patients agree to share information with their physicians, it will allow to monitor patients' status regularly. This is relevant as physicians are only able to know patients' status when they go to the medical centres for regular visits (twice a year) or for emergencies [14]. Personalised context-aware solutions have the potential to provide meaningful data to be used as input for more complex decision-making process including technologies related to big and small data. Furthermore, the strategic involvement of carers in the communication process has the potential to enhance preventive and reactive actions. The research provides a more comprehensive way of framing the context of a patient with the aim of enhancing decision-making in the asthma management process.

Some future directions of the research are: assessing the prototype by users, implementing Case-Base Reasoning to detect abnormalities and emergencies, and creating a Virtual Assistant to support tasks. These features will be built on top of the personalisation and context-awareness layers. 


\section{References}

1. Global Initiative for Asthma: Global Strategy for Asthma Management and Prevention. www.ginasthma.org (2017). Accessed 31 Jan 2018

2. Asthma UK: What is Asthma? www.asthma.org.uk. Accessed 30 Jan 2018

3. Asthma Australia: Understanding asthma. www.asthmaaustralia.org.au. Accessed 30 Jan 2018

4. NHS: Asthma-NHS.UK. www.nhs.uk/Conditions/Asthma. Accessed 30 Jan 2018

5. Center for Disease Control and Prevention: CDC - Asthma Surveillance Data. https://www.cdc.gov/asthma/asthmadata.htm. Accessed 30 Jan 2018

6. Asthma UK: Annual Report \& Accounts. www.asthma.org.uk. Accessed 30 Jan 2018

7. Dey, A.K.: Understanding and using context. Pers. Ubiquit. Comput. 5(1), 4-7 (2001). https://doi.org/10.1007/s007790170019

8. Augusto, J.C., et al.: Intelligent environments: a manifesto. Hum. Centric Comput. Inf. Sci. 3, 12 (2013). https://doi.org/10.1186/2192-1962-3-12

9. Teixeira, A., et al.: Design and development of Medication Assistant: older adults centred design to go beyond simple medication reminders. Univ. Access Inf. Soc. 16(3), 545-560 (2017). https://doi.org/10.1007/s10209-016-0487-7

10. Tavares, J., et al.: Hefestos: an intelligent system applied to ubiquitous accessibility. Univ. Access Inf. Soc. 15(4), 589-607 (2016). https://doi.org/10.1007/s10209-0150423-2

11. Paredes, H., et al.: SOSPhone: a mobile application for emergency calls. Univ. Access Inf. Soc. 13, 277-290 (2014). https://doi.org/10.1007/s10209-013-0318-z

12. Wagner, S., Toftegaard, T.S., Bertelsen, O.W.: Increased data quality in home blood pressure monitoring through context awareness. In: 5th International Conference on Pervasive Computing Technologies for Healthcare PervasiveHealth and Workshops, pp. 234-237 (2011). https://doi.org/10.4108/icst.pervasivehealth. 2011.245968

13. Chang, S., Chiang, R., Wu, S.: A Context-Aware, Interactive M-Health System for Diabetics. IT Prof. 18(3), 14-22 (2016). https://doi.org/10.1109/MITP.2016.48

14. Osuntogun, A.A., Arriaga, R.I.: Physician usage of technology and opportunities for continuous care management of pediatric Asthma patients. In: 4th International Conference on Pervasive Computing Technologies for Healthcare, pp. 1-6 (2010). https://doi.org/10.4108/ICST.PERVASIVEHEALTH2010.8868

15. Yun, T.J., et al.: Assessing Asthma management practices through in-home technology probes. In: 2010 4th International Conference on Pervasive Computing Technologies for Healthcare, pp. 1-9 (2010). https://doi.org/10.4108/ICST. PERVASIVEHEALTH2010.8839

16. Al-Dowaihi, D., et al.: MBreath: Asthma monitoring system on the go. In: IEEE ICCMA 2013, pp. 1-4 (2013). https://doi.org/10.1109/ICCMA.2013.6506169

17. Uwaoma, C., Mansingh, G.: Towards real-time monitoring and detection of Asthma symptoms on resource-constraint mobile device. In: Annual IEEE CCNC 2015, pp. 47-52 (2015). https://doi.org/10.1109/CCNC.2015.7157945

18. Kwan, A.M., et al.: Personal lung function monitoring devices for Asthma patients. IEEE Sens. J. 15(4), 2238-2247 (2015). https://doi.org/10.1109/JSEN. 2014.2373134

19. Anantharam, P., et al.: Knowledge-driven personalized contextual mHealth service for Asthma management in children. In: 2015 IEEE International Conference on Mobile Services, pp. 284-291 (2015). https://doi.org/10.1109/MobServ.2015.48 
20. Ra, H.-K., et al.: AsthmaGuide: an Asthma monitoring and advice ecosystem. In: 2016 IEEE Wireless Health, pp. 128-135 (2016). https://doi.org/10.1109/WH. 2016.7764567

21. Dieffenderfer, J., et al.: Low power wearable systems for continuous monitoring of environment and health for chronic respiratory disease. IEEE J. Biomed. Health Inform. 20(5), 1251-1264 (2016). https://doi.org/10.1109/JBHI.2016.2573286

22. Augusto, J.C., et al.: The user-centred intelligent environments development process as a guide to co-create smart technology for people with special needs. Univ. Access Inf. Soc. 17(1), 115-130 (2018). https://doi.org/10.1007/s10209-016-05148 
Open Access This chapter is licensed under the terms of the Creative Commons Attribution 4.0 International License (http://creativecommons.org/licenses/by/4.0/), which permits use, sharing, adaptation, distribution and reproduction in any medium or format, as long as you give appropriate credit to the original author(s) and the source, provide a link to the Creative Commons license and indicate if changes were made.

The images or other third party material in this chapter are included in the chapter's Creative Commons license, unless indicated otherwise in a credit line to the material. If material is not included in the chapter's Creative Commons license and your intended use is not permitted by statutory regulation or exceeds the permitted use, you will need to obtain permission directly from the copyright holder. 